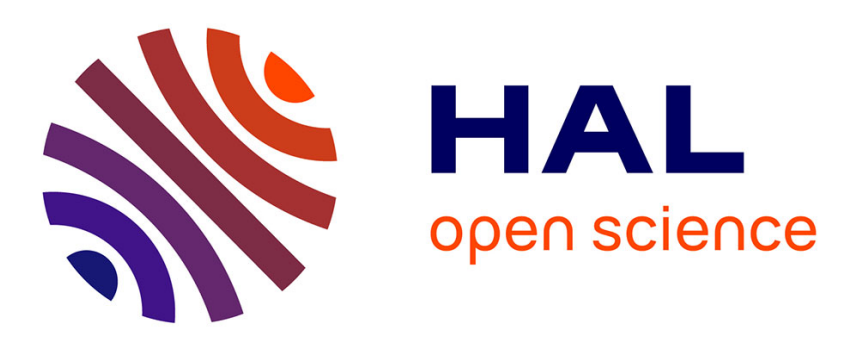

\title{
Towards fast, generic video inpainting
}

Alasdair Newson, Matthieu Fradet, Patrick Pérez, Andrés Almansa, Yann

Gousseau

\section{To cite this version:}

Alasdair Newson, Matthieu Fradet, Patrick Pérez, Andrés Almansa, Yann Gousseau. Towards fast, generic video inpainting. Proceedings of the 10th European Conference on Visual Media Production - CVMP '13, ACM SIGGRAPH, Nov 2013, London, United Kingdom. 10.1145/2534008.2534019 . hal-00838927

\section{HAL Id: hal-00838927 \\ https://hal.science/hal-00838927}

Submitted on 27 Jun 2013

HAL is a multi-disciplinary open access archive for the deposit and dissemination of scientific research documents, whether they are published or not. The documents may come from teaching and research institutions in France or abroad, or from public or private research centers.
L'archive ouverte pluridisciplinaire HAL, est destinée au dépôt et à la diffusion de documents scientifiques de niveau recherche, publiés ou non, émanant des établissements d'enseignement et de recherche français ou étrangers, des laboratoires publics ou privés. 


\section{Towards fast, generic video inpainting}

\author{
Alasdair Newson, Matthieu Fradet, \\ Patrick Pérez \\ Technicolor \\ 1 Avenue Belle Fontaine \\ 35 , Cesson-Sévigné \\ alasdair.newson@technicolor.com \\ matthieu.fradet@technicolor.com \\ patrick.perez@technicolor.com
}

\author{
Andrés Almansa, Yann Gousseau \\ Télécom ParisTech - LTCI CNRS \\ 43 rue Barrault \\ 75013 Paris \\ andres.almansa@telecom-paristech.fr \\ yann.gousseau@telecom-paristech.fr
}

\begin{abstract}
Achieving globally coherent video inpainting results in reasonable time and in an automated manner is still an open problem. In this paper, we build on the seminal work by Wexler et al. to propose an automatic video inpainting algorithm yielding convincing results in greatly reduced computational times. We extend the PatchMatch algorithm to the spatio-temporal case in order to accelerate the search for approximate nearest neighbours in the patch space. We also provide a simple and fast solution to the well known over-smoothing problem resulting from the averaging of patches. Furthermore, we show that results similar to those of a supervised state-of-the-art method may be obtained on high resolution videos without any manual intervention. Our results indicate that globally coherent patch-based algorithms are feasible and an attractive solution to the difficult problem of video inpainting.
\end{abstract}

\section{Keywords}

Video inpainting, PatchMatch, exemplar-based, patches, spacetime occlusion

\section{INTRODUCTION AND PRIOR WORK}

The goal of "image inpainting" [1] is to convincingly replace or "disocclude" $[2,3]$ a region with some other image content. The vast majority of inpainting methods find this information in the image itself. One of the main difficulties of this task is the correct reproduction of both geometric structures and textures. Video inpainting is the problem of inpainting a spatio-temporal hole in a video. This adds new technical challenges such as inpainting foreground, background and moving objects. Additionally, the execution time becomes a critical aspect, as certain video inpainting algorithms which do not deal with this aspect specifically may take days or even weeks to execute.

Generally speaking, video inpainting algorithms belong to either the "object-based" or "patch-based" category. Object-based algorithms usually segment the video into moving foreground and back- ground that is either still or displays simple motion. These segmented image sequences are then inpainted using separate algorithms. The background is often inpainted using image inpainting methods such as [4], whereas moving objects are often copied into the occlusion as smoothly as possible. Unfortunately, such methods often include restrictive hypotheses on the moving objects' motion, such as strict periodicity. Some object-based methods include [5, $6,7,8]$.

Patch-based methods are based on the intuitive idea of copying and pasting small video "patches" (rectangular cuboids of video information) into the occluded area. The first patch-based method to ensure temporal coherency in video inpainting was described by Wexler et al. in [9]. This is an iterative method that may be seen as a heuristic to solve a global optimisation problem. The high dimensionality of the problem makes the algorithm very slow, requiring up to several days for a few seconds of VGA video. Patwardhan et al. [5] also use a patch-based approach. This is a greedy algorithm, and therefore cannot guarantee global coherency.

Pritch et al. [10] first proposed to use the discrete optimisation algorithm called "graph cuts" [11] for the purpose of image inpainting. This method optimises an energy functional of the shift map to inpaint an image. The shift map is the mapping from the set of pixels in the occlusion to a subset of the unoccluded pixels, which corresponds to the nearest neighbours from a patch-based point of view. Other image inpainting methods such as $[12,13]$ have also used this sort of strategy. The idea of using graph cuts for video inpainting was recently introduced by Granados et al. in [14]. They propose a semi-automatic algorithm which optimises the spatio-temporal shift map. This algorithm presents impressive results on higher resolution images than are previously found in the literature (up to $1120 \times 754$ pixels). However, in order to reduce the large search space and high time complexity of the optimisation method, manual tracking of moving occluded objects is required. To the best of our knowledge, the inpainting results of Granados et $a l$. are the most convincing to date, and we shall therefore compare our algorithm with these results.

Another recent method by Facciolo et al. [15] deals with video editing in the gradient domain. However, the goal of this method is to enforce temporal consistency given a certain inpainting result, rather than producing the actual video inpainting result. Nevertheless, such post-processing methods could be used to refine the results of video inpainting algorithms.

The seminal work of Wexler et al. [9] is widely cited and well- 
known in the video inpainting domain, mainly because it ensures global coherency in an automatic manner. Inpainting algorithms as recent as [16] have presented variations of this work for image inpainting purposes. However, due to extremely long execution times, it is difficult to implement and experiment with, making the setting of implementation details and parameters especially tedious. This is in fact the greatest obstacle preventing the progress of research in this direction. In this work, we wish to produce an algorithm which is able to achieve the global coherence of the algorithm presented in [17], while maintaining execution times which are not prohibitive for experimentation and practical use. For this, we build on Wexler's central idea of iterative aggregation of nearest neighbours in the patch space to obtain an automatic algorithm with greatly reduced execution times. We extend the PatchMatch algorithm to the spatio-temporal domain in order to accelerate the search for approximate nearest neighbours. Furthermore, we propose a simple solution to the well-known over-smoothing problem due to the averaging of patches, and also provide specific implementation details to make our work reproducible. The resulting algorithm yields similar results to the original one, with a speedup of up to 50 times, and, furthermore, is able to successfully inpaint high resolution videos in an automatic manner, which has not been done before. We reduce execution times by an order of magnitude in comparison with the most recent supervised method [14], with visually similar results.

\section{PATCH-BASED GLOBAL OPTIMISATION}

The generic video inpainting problem is that of reconstructing the content of a spatio-temporal hole (occlusion) in a video. Our video inpainting approach builds on the foundations laid out by Wexler $e t$ al. in [17]. This algorithm fills a spatio-temporal volume using the information in the unoccluded parts of the video. The solution is obtained by the minimisation of a global patch-based functional. To achieve this, the algorithm alternates between the search for the nearest neighbours (NNs) of spatio-temporal patches in the occluded region and the reconstruction of the inpainted volume using these NNs. This process is iterated several times in order to converge to a solution. As in many optimisation problems, a multi-resolution spatio-temporal pyramid is used in order to avoid local minima. We shall now present the algorithm of [17] in greater detail.

\subsection{The Space-Time Completion algorithm}

As far as possible, we retain the notation found in [17]. Let $\mathcal{H}$ be the spatio-temporal occlusion and $\mathcal{D}$ the data set (unoccluded area). Furthermore, let $\tilde{\mathcal{D}}$ be the region in which all patches are completely outside of $\mathcal{D}$. The ANN search is restricted to patches belonging to $\tilde{\mathcal{D}}$. Let $p=(x, y, t)$ be a position in the video and $W_{p}$ a patch centred on $p$. Let us also define the NN shift map $\phi: \mathbb{R}^{2} \rightarrow \mathbb{R}^{2}$, which indicates that the NN (in terms of a given patch distance) of $W_{p}$ is centred at the position $p+\phi(p)$. We define this NN patch as $W_{p+\phi(p)}$. Let $\mathcal{N}_{p}$ be the spatio-temporal positions in the patch centred on $p$. In the original paper, Wexler $e t$ $a l$. use $5 \times 5 \times 5$ patches, with each position in a patch containing the following vector of patch components : $(R, G, B, \beta u, \beta v)$, where $R, G$ and $B$ are the colour values, $u$ and $v$ are roughly estimated optical flow components, and $\beta$ is a scaling factor. The distance $d\left(W_{p}, W_{p+\phi(p)}\right)$ is the sum of squared differences (SSD) of each of the patch components.

The original algorithm of Wexler et al. contains three main components:
- The search for NNs

- The reconstruction of a video using the NNs

- The implementation of the spatio-temporal pyramid.

As previously mentioned, the first two steps are iterated several times, at each pyramid level.

An exhaustive search for exact NNs is obviously far too timeconsuming. Therefore, in order to make the algorithm feasible, the work of Arya et al. [18] is used in [17] to find approximate nearest neighbours (ANNs), rather than the exact nearest neighbours.

Concerning the reconstruction of the solution, Wexler et al. propose a scheme in which the colour value $c$ of a pixel $p \in \mathcal{H}$ is obtained with a weighted mean of all the colours given by the ANNs of the patches which contain $p$ :

$$
c_{p}=\frac{\sum_{i \in \mathcal{N}_{p}} \alpha_{p}^{i} s_{p}^{i} c^{i}}{\sum_{i \in \mathcal{N}_{p}} \alpha_{p}^{i} s_{p}^{i}}
$$

with

$$
s_{p}^{i}=\exp -\frac{d\left(W_{i}, W_{i+\phi(i)}\right)}{2 \sigma^{2}}
$$

where $c^{i}$ is the colour value in the ANN of $W_{i}$ at the position corresponding to $p$. The value $\alpha_{p}^{i}$ is a weight given to the pixel $i$ which reflects the distance of $i$ to the occlusion boundary. More precisely, $\alpha_{p}^{i}=\gamma^{\operatorname{dist}(p)}$, where $\operatorname{dist}(p)$ is the distance from $p$ to the occlusion boundary, and $\gamma$ is set to 1.3. Finally, $\sigma$ is defined as the 75th percentile of all the distances $d\left(W_{i}, W_{i+\phi(i)}\right), i \in \mathcal{N}_{p}$. One drawback of the previous weighted mean is a blurred inpainting result. Therefore, Wexler et al. propose another reconstruction method which is a robust estimation of the value of $c_{p}$ using the mean-shift algorithm. This mean-shift is carried out on points in the RGB colour space, provided by $c^{i}$. Each point is weighted by $\alpha_{p}^{i} s_{p}^{i}$, and the dominant mode is found in this space. During the algorithm, the bandwidth used for the mean shift is gradually reduced. The goal of this scheme is to gradually decide on the best ANN for each $W_{p}$. The mean shift algorithm is used in order to make the reconstruction robust to poor ANNs found by the ANN search, and also reduces the blurring effect in the final result.

Finally Wexler et al. use a spatio-temporal Gaussian pyramid in order to improve the optimisation results, as is common in inpainting algorithms. The downsampling scheme is not precisely defined in [17]. The upsampling of a solution from one level to another is done by propagating the current ANNs to the finer level. The finer solution is then produced with the same reconstruction scheme, using the new ANNs.

We now proceed to explain the details of the proposed algorithm.

\subsection{Approximate nearest neighbour search}

The search for ANNs is the most time-consuming part of the algorithm, and thus the most important to work on to obtain faster execution times. Quite recently, a very efficient ANN search algorithm named "PatchMatch" was proposed by Barnes et al. [19], specifically for the task of finding perceptually close patches 
"Beach Umbrella"
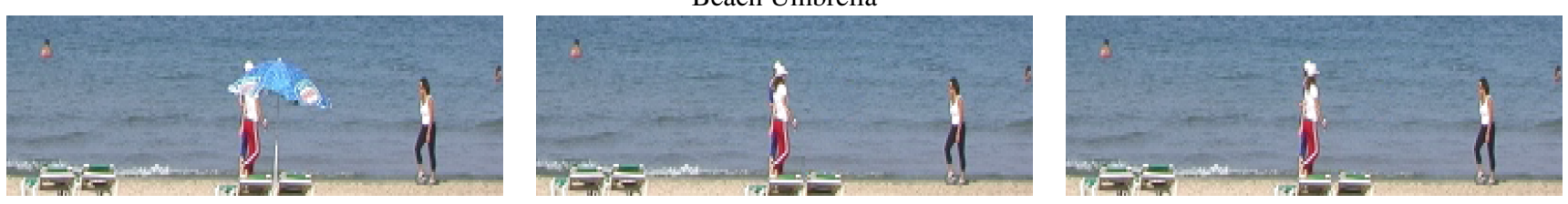

"Crossing Ladies"
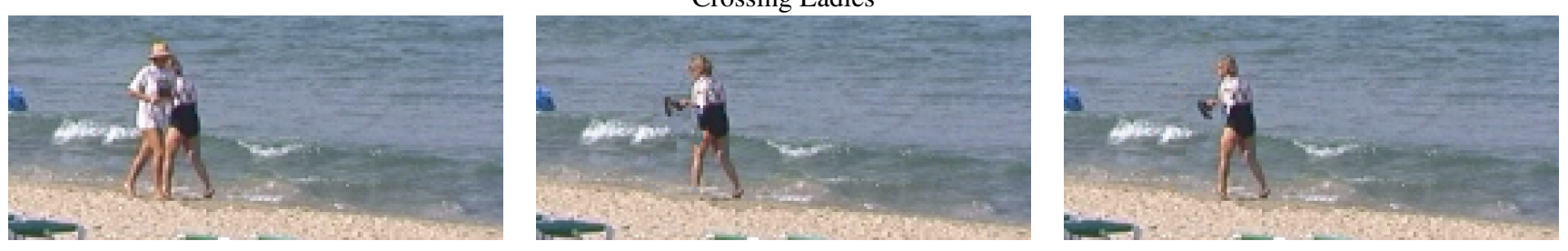

"Jumping girl"

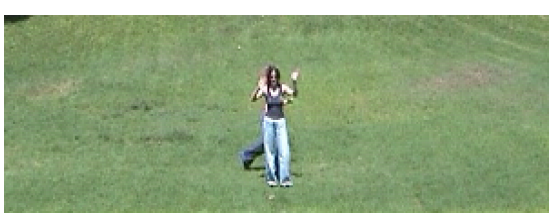

Original frames

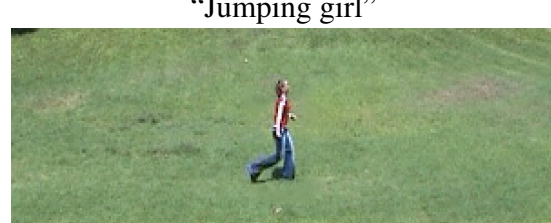

Inpainting result from [17]

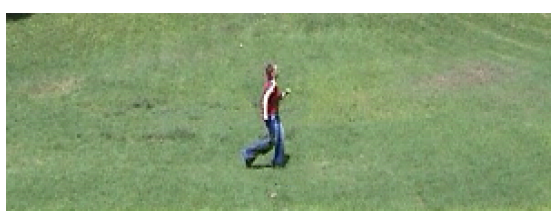

Our inpainting result

Figure 1: Comparison of our results with the those of [17]. Visually, the obtained inpainted is very similar, but we are able to reduce the ANN search time by a factor of up to 50 times.

in images. PatchMatch's efficiency comes from the observation that ANN shift maps are often very regular between images (in fact they are piecewise constant), if the same image content is present. Barnes et al. suggested the use of PatchMatch for image inpainting purposes, and this algorithm was subsequently used for this purpose in the "Content Aware Fill" tool by Photoshop CS5 [20], and more recently by Darabi et al. [16]. To the best of our knowledge it has never been proposed for video inpainting. We propose to extend the PatchMatch algorithm to the spatio-temporal setting, in order to reduce the extremely long search times obtained with the ANN search described in [18]. It seems quite natural to use PatchMatch for video inpainting, since the piecewise constancy of image ANN shift maps should work well in the present case, since the image content is repeated throughout the video.

The original PatchMatch contains three steps : initialisation, propagation and random search. The initialisation is done by randomly associating an ANN to each patch $W_{p}$, with $p=(x, y)$. During propagation, the patches are sequentially scanned from low to high indices, first in the $x$ and then in the $y$ dimension. For a given patch $W_{x, y}$, the algorithm considers the following ANNs : $W_{(x, y)+\phi(x-1, y)}$ and $W_{(x, y)+\phi(x, y-1)}$. If one of these ANNs has a smaller patch distance with respect to $W_{(x, y)}$ than $W_{(x, y)+\phi(x, y)}$, then $W_{(x, y)+\phi(x, y)}$ is replaced with the best new ANN. The scanning order is reversed for the next iteration of the propagation (from high to low), and the algorithm tests $W_{(x, y)+\phi(x+1, y)}$ and $W_{(x, y)+\phi(x, y+1)}$. In the two different scanning orderings, the important point is obviously to use the neighbours which have already been tested in the current propagation step. The motivation for this step is the idea that objects in images are spatially coherent, and therefore that offsets which lead to good ANNs for a given patch are likely to produce good ANNs in the proximity of this patch. The third step, the random search, consists of randomly looking for better ANNs of each $W_{p}$ in an increasingly small area around $W_{p+\phi(p)}$, starting with a maximum search distance. The random ANNs are centred at the following positions :

$$
q_{j}=p+\phi(p)+w \rho^{j} \mathbf{R}_{j},
$$

for all $j \in \mathbb{N}$ such that $w \rho^{j}>1$, where $\mathrm{w}$ is the maximum search radius around the $W_{p+\phi(p)}, \mathbf{R}_{i}$ is a pair of random variables in $[-1,1] \times[-1,1]$ and $\rho$ is the reduction factor of the search window size. In the original PatchMatch, $\rho$ is set to 0.5 . This random search avoids the algorithm getting stuck in local minima.

We now detail our generalisation of PatchMatch to the video setting. Let $W_{(x, y, t)}$ be a spatio-temporal patch centred at $(x, y, t)$. We initialise the ANNs randomly in any position $p \in \tilde{\mathcal{D}}$. We redefine $\phi$ as the spatio-temporal ANN offset map : $\phi: \mathbb{R}^{3} \rightarrow \mathbb{R}^{3}$.

For propagation, we need to define a scanning order of the patches. As in [19], we scan in the $x$ dimension and then the $y$ dimension. Lastly, we scan in the temporal dimension. For each $W_{(x, y, t)}$, we test $W_{(x, y, t)+\phi(x-1, y, t)}, W_{(x, y, t)+\phi(x, y-1, t)}$ and $W_{(x, y, t)+\phi(x, y, t-1)}$. As in [19], between propagation iterations the scanning order is reversed, and $W_{(x, y, t)+\phi(x+1, y, t)}, W_{(x, y, t)+\phi(x, y+1, t)}$ and $W_{(x, y, t)+\phi(x, y, t+1)}$ are tested. For this propagation step, it is important to know whether the scanning order of the video dimensions has any influence on the quality of the resulting ANNs. In particular, the regularity properties (upon which the PatchMatch algorithm is based) may be different for different dimensions. For example, if we suppose that there is greater information redundancy along the $t$ axis, then it is preferable to analyse the ANNs along this axis before analysing the others. This sort of consideration was not taken into account in [19], since there is no reason a priori to suppose that the $x$ and $y$ axes present different degrees of coherence. For a visual illustration 


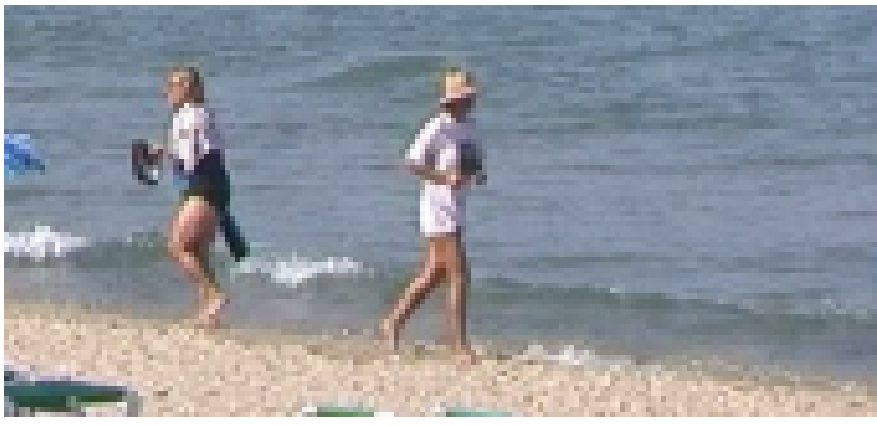

Original frame : "Crossing ladies"

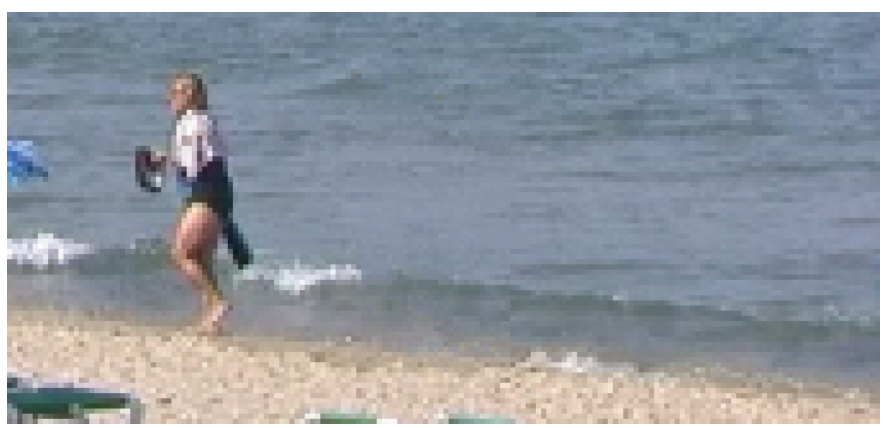

Mean shift

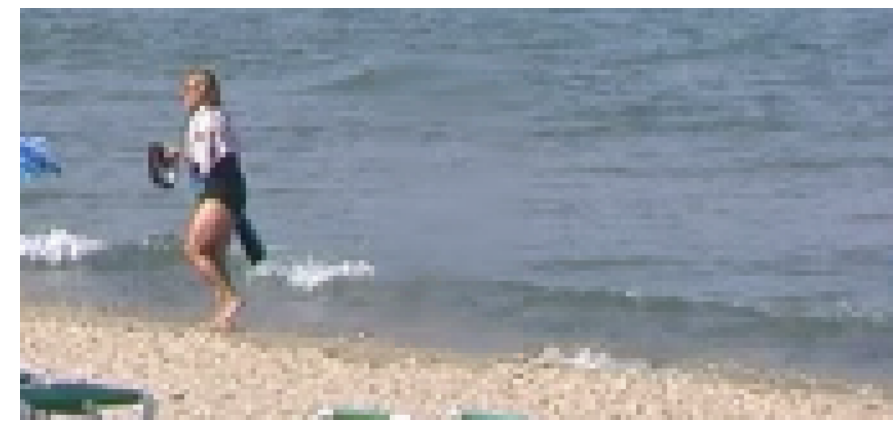

Weighted average

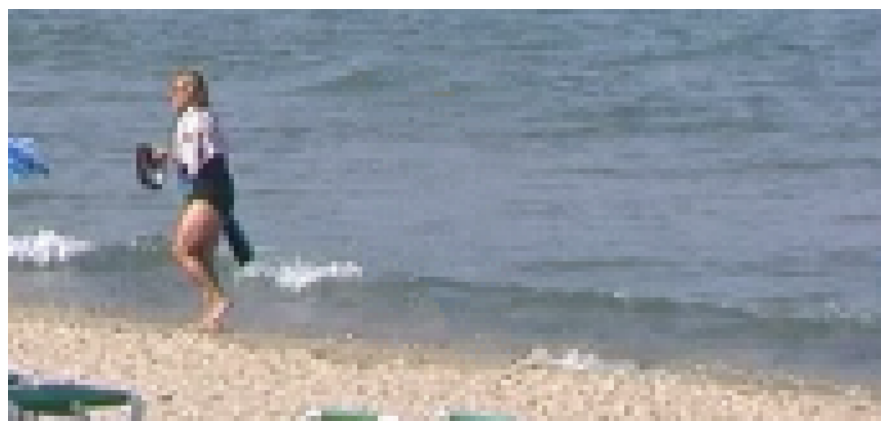

Best patch

Figure 2: Comparison of different reconstruction methods. We observe that the reconstruction using the best patch at the end of the algorithm produces similar results to the use of the mean shift algorithm. Please note that the blurring effect is best viewed in the pdf version of the paper.

of the neighbours used during the propagation step, see Figure 3.

Table 1 compares the average ANN error (in terms of the sum of square differences) per patch component, for all the scanning orderings (nine in total). The three standard sequences of Wexler et al. [17] are analysed in this manner. We observe that the patch error is very stable with respect to the scanning order. Therefore, we have chosen (arbitrarily) to maintain the original scanning order of PatchMatch, and add the $t$ dimension afterwards.

The extension of the random search step is straightforward. Instead of searching in a square around current ANNs, we search in a cube, for all $p \in \mathcal{H}$. We keep the original window size reduction parameter $\beta=0.5$. In the current work, we allow the random search to search the entire video, however this could possibly be tuned to accelerate the algorithm further. We set the number of iterations of propagation and random search to 10 , which gives good results on all the videos we tested. Between iterations of ANN search and reconstruction, we initialise PatchMatch with the previous ANNs.

In [17], the patches include rough estimations of an optical flow, $u$ and $v$. Having experimented with and without these elements, we found that their influence was very small. Therefore, we only use the colour components in our patches.

\subsection{Reconstruction}

As mentioned above, two reconstruction schemes are presented in [17]: weighted averaging and a robust mean shift colour estimation. While the mean shift has advantages such as avoiding blurring in the final results, it complicates the algorithm and adds new parameters, such as the speed at which the mean shift's band width is reduced, for which details are not given in [17].

Dealing with this blurring problem is particularly important if video textures are present, such as the waves in the "Beach Umbrella" and "Crossing Ladies" examples of Wexler et al. An important question is whether the gradual reduction of the band-width of the mean shift throughout the algorithm is necessary, or if this may be delayed until the end of the algorithm. In terms of Equation (1), the mean shift band-width reduction is very similar to reducing the parameter $\sigma$ throughout the iterations of ANN search and reconstruction.

After testing and comparing various reconstruction schemes, we propose the following method. Throughout the algorithm we use the weighted mean of Equation (1). Then, after convergence of this scheme at the finest pyramid level, we reconstruct the video using the $W_{i \in \mathcal{N}_{p}}$ with the smallest distance for each $W_{p}$. This reconstruction correctly "deblurs" the result, and is far simpler to implement and use than the mean shift. With respect to execution time, the mean shift algorithm has a time complexity of $O\left(T\left|W_{p}\right|^{2} n_{o}\right)$, where $T$ is the number of iterations of each mean shift, $\left|W_{p}\right|$ is the size of a patch, and $n_{o}$ is the number of occluded pixels. This mean shift is repeated at each iteration within each pyramid level. The proposed reconstruction, on the other hand, has a time complexity of $O\left(\left|W_{p}\right| n_{o}\right)$, the same as the weighted mean. Even though the ANN search represents the majority of computation time, it is obviously preferable to use a reconstruction scheme which is linear, rather than quadratic, with respect to the patch size.

Figure 2 shows some visual comparisons of the inpainting results 


\begin{tabular}{|l|c|c|c|c|c|c|c|}
\hline \multirow{2}{*}{ Propagation order } & \multicolumn{6}{|c|}{ Mean ANN patch error, per component } \\
\cline { 2 - 8 } & $\mathrm{t}, \mathrm{y}, \mathrm{x}$ & $\mathrm{y}, \mathrm{t}, \mathrm{x}$ & $\mathrm{t}, \mathrm{x}, \mathrm{y}$ & $\mathrm{x}, \mathrm{t}, \mathrm{y}$ & $\mathrm{y}, \mathrm{x}, \mathrm{t}$ & $\mathrm{x}, \mathrm{y}, \mathrm{t}$ & Full search \\
\hline Beach Umbrella & 9.22 & 9.61 & 9.49 & 9.54 & 9.57 & 9.11 & 6.83 \\
Crossing Ladies & 7.53 & 7.44 & 7.42 & 7.51 & 7.50 & 7.35 & 6.14 \\
Jumping Girl & 6.48 & 6.52 & 6.40 & 6.49 & 6.50 & 6.45 & 4.80 \\
\hline
\end{tabular}

Table 1: Comparison of propagation scanning ordering, in terms of average component error between a patch and its approximate nearest neighbour. On the last row is the average error of the true nearest neighbour.

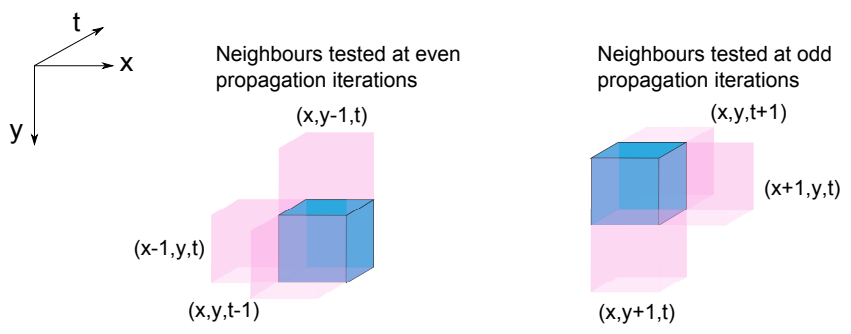

Figure 3: A visual illustration of the neighbour whose shift maps are tested during the propagation step of the spatio-temporal PatchMatch. PatchMatch changes the neighbours used for testing between odd and even iterations.

using different reconstruction schemes. The weighted mean, the mean shift and the proposed reconstruction are compared. We observe that the reconstruction using the best ANN (the proposed reconstruction) is extremely similar to the result using the mean shift. In this particular case, the use of the best patch at the end of the algorithm corresponds to choosing one amongst a set of patches which are randomly distributed around an average value. This, in effect, recreates the "noise" which corresponds to the video texture of the sea.

Another crucial part of the reconstruction is the initialisation of the inpainting solution at the coarsest pyramid resolution. This initialisation is left unspecified in [17]. To initialise the solution, we inpaint at the coarsest level using an onion peel approach, with a layer thickness of one pixel. The pixels within a given layer of the occlusion are inpainted in parallel, using Equation (1). Since the information available in each patch on the border of the occlusion is incomplete, we only compare the unoccluded pixels of a patch during the ANN search. Once these ANNs are found, we must choose which one should be used for reconstruction. Some will be quite unreliable, as only a small part of the patches are compared. In our implementation, we only use the ANNs of patches whose centres are located outside the current occlusion layer. For a spherical occlusion, this means that roughly half of the available patches are used.

Finally, in [17] the number of iterations in a pyramid level is fixed. In the interest of robustness, we use the average pixel colour difference in each channel between iterations as a stopping criterion. If this falls below a certain threshold, we stop the iteration at the current level. We set this threshold to 0.1.

\subsection{Spatio-temporal subsampling}

The last part of the algorithm concerns the implementation of the multi-resolution spatio-temporal pyramid. As noted in [14], temporal subsampling may produce undesirable effects. We do not temporally subsample either, apart from cases where objects are occluded for a long time, which was done on only one video
("Jumping girl") in this paper. Before downsampling, we filter the video by averaging $2 \times 2$ image blocks.

The use of a spatio-temporal pyramid implies two further details: the upsampling of the current solution and whether or not to pass an upsampled version of the current ANNs as an initialisation to the PatchMatch at the finer level. We keep the same solution upsampling procedure as in [17], which is able to preserve fine details. However, we do not initialise PatchMatch at a finer level using the coarse level ANNs. This avoids biasing the finer ANNs towards certain spatio-temporal positions. Since certain details may not be visible at coarser levels, we prefer to let PatchMatch find the best ANNs at each level independently.

\section{RESULTS}

Since the main goal of our work is to achieve generic video inpainting with good results in reduced time, we evaluate our algorithm in terms of both execution time and visual results. In this section, we shall compare our work to that of Wexler et al. [17] and the most recent video inpainting method of Granados et al. [14].

In all of our experiments, we have retained the parameters as they are presented in the main body of the paper. In particular, we keep the patch size of $5 \times 5 \times 5$ as used in [17]. We use ten iterations of propagation/random search during the PatchMatch algorithm and set the window size reduction factor $\beta$ to 0.5 . During the construction of the spatio-temporal pyramid, we do not subsample temporally in any of the videos, apart from "Jumping girl".

In [17], neither the initialisation of the occluded area nor the number of iterations per pyramid level are precisely defined. Since total execution time greatly depends on these details, we shall only compare our algorithm to Wexler's in terms of ANN search time. This represents the majority of total time (in [17], it takes up 95 percent of execution time), thus it makes sense to compare these timings. While our reconstruction scheme also reduces execution time, it will not represent as great a speed-up as the ANN search time, so we do not compare reconstruction timings. We also provide our total execution times, for reference.

Comparisons with Granados's methods are more difficult, as it is semi-automatic. However, several execution times are provided in [14] and we compare our timings to these.

We recall that Wexler uses the method of [18] to search for ANNs. This method uses a parameter, $\varepsilon$, which determines the accuracy of the ANNs. As this parameter is not given in [17], we set it to produce the same average error per component as the spatiotemporal PatchMatch. This is the same evaluation method used by Barnes et al. in [19]. Specifically, we set $\varepsilon$ to 10. In Table 2, the ANN search time comparisons may be seen. We obtain a speedup of 20-50 times over the method of [18]. 

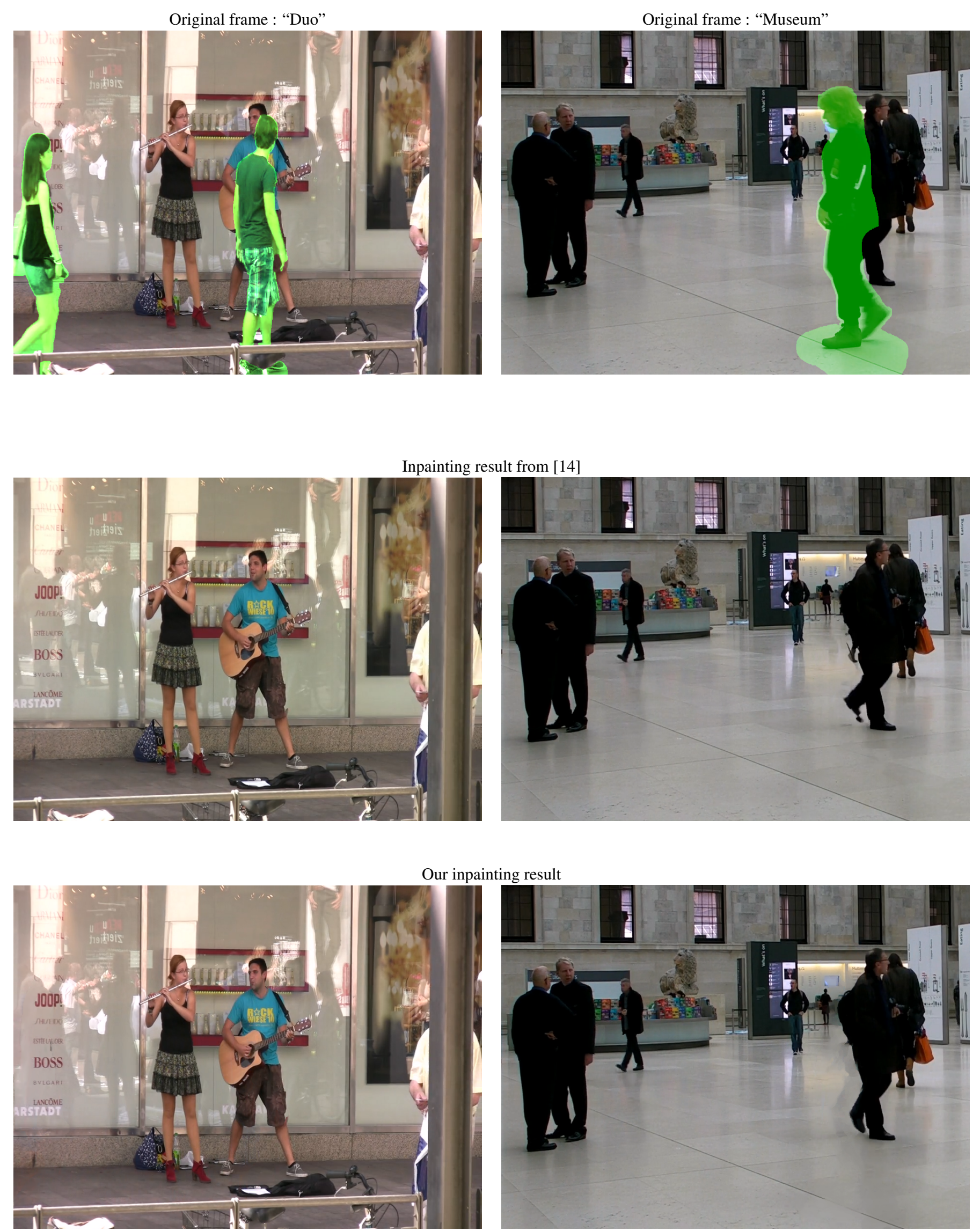

Figure 4: We achieve similar results to those of [14] in an order of magnitude less time, without user intervention. The occlusion masks are highlighted in green. Result videos are viewable at http://www.enst.fr/ gousseau/videoinpainting 


\begin{tabular}{|c|c|c|c|c|c|}
\hline \multirow{2}{*}{ Algorithm } & \multicolumn{5}{|c|}{ Approximate nearest neighbour execution times, for all occluded pixels at full resolution. } \\
\cline { 2 - 6 } & $\begin{array}{c}\text { Beach Umbrella } \\
264 \times 68 \times 200\end{array}$ & $\begin{array}{c}\text { Crossing Ladies } \\
170 \times 80 \times 87\end{array}$ & $\begin{array}{c}\text { Jumping Girl } \\
\text { 300x100x239 }\end{array}$ & $\begin{array}{c}\text { Duo } \\
960 \times 704 \times 154\end{array}$ & $\begin{array}{c}\text { Museum } \\
1120 \times 754 \times 200\end{array}$ \\
\hline Wexler & $985 \mathrm{~s}$ & $942 \mathrm{~s}$ & $7877 \mathrm{~s}$ & - & - \\
Ours & $50 \mathrm{~s}$ & $28 \mathrm{~s}$ & $155 \mathrm{~s}$ & $2515 \mathrm{~s}$ & $3958 \mathrm{~s}$ \\
\hline \hline Algorithm & \multicolumn{5}{|c|}{ Total execution time } \\
\hline Granados & 11 hours & - & - & - & 90 hours \\
Ours & 21 mins & 6 mins & 62 mins & 7.1 hours & 8.64 hours \\
\hline
\end{tabular}

Table 2: Partial and total inpainting execution times on different examples. The partial inpainting times represent the time taken for the ANN search for all occluded patches at the full resolution. Note that for the "museum" example, Granados's algorithm is parallelised over the different occluded objects and the background, whereas ours is not.

In comparison to the work of [14] our method took $8 \mathrm{~h} 38 \mathrm{~m}$ on the longest example ("Museum"), while Granados et al. report a total execution time of 90 hours, with a similar computer architecture to ours. We used a 64-bit machine with a $2.67 \mathrm{GHz}$ Intel Xeon processor. For fairness, only one core of the processor was used (as in [14]). However, in Granados's work each occluded object and the background are inpainted separately, in parallel, whereas we treat all objects simultaneously. In the "Museum" example Granados reports [21] seven objects plus the background, meaning that the real workload is several times greater.

We now compare the time complexity of our algorithm with that of [21]. The patch match algorithm runs in $O\left(6 Q n_{o} d_{p}+Q n_{o} \log _{2}(N)\right)$ time, where (as above) $n_{o}$ is the number of occluded pixels, $d_{p}$ is the dimension of the patches, $N$ is the maximum random search space size, and $Q$ is the number of iterations of propagation/random search. The reconstruction runs in $O\left(n_{o} d_{p}\right)$ time. In comparison, Granados et al. report a time complexity of $O\left(n_{o}^{3} N\right)$. This explains the quite long execution times reported in [14] and in particular the need to restrict the search space manually.

It is interesting to note that the search space restriction used in [14] may not only reduce execution times, but might in fact improve inpainting results. Liu et al. [13], who used graph cuts for the purpose of image inpainting, have reported that inpainting results are often degraded when the entire image is used as a search space. In the case of image inpainting, restricting the search space is not a significant problem, since in the vast majority of cases the necessary information is situated around the occlusion. On the other hand, this is not the case for video inpainting. For example, if an object displays periodic motion with a particularly large period, then the video information may be situated at a correspondingly large spatio-temporal distance. In the proposed algorithm, we do not restrict the search space and we are able to produce coherent results. Finally, in the case of graph cut based inpainting schemes such as $[10,13,14]$, it is not possible to use patches of arbitrary size, due to execution time issues. This is an extremely limiting factor, and is a strong argument in favour of inpainting schemes such as that which have presented in this work.

Figure 1 and Figure 4 present visual comparisons of our results with those of [17] and the high resolution results of [14]. We observe that our results are qualitatively very similar. These results were obtained in highly reduced execution times, and in a generic manner, without manual user intervention, validating the initial goal of our work. The complete comparison videos may be viewed at the following address : http://www.enst.fr/ gousseau/videoinpainting.

\section{FURTHER WORK}

Though we have shown that automatic generic video inpainting with reduced execution times is possible, there are several points in the proposed algorithm which could be improved upon. In particular, the use of a spatio-temporal pyramid means that certain comparisons may be ambiguous at coarse resolutions. Liu et al. [13] introduce new components in the patch comparison to avoid this problem, and this could possibly be extended to the video context. Furthermore, we have not exploited the parallel aspect of both the random search step and the reconstruction. A parallel implementation of these steps could further decrease the execution times, making results available more quickly and increasing experimental possibilities. Finally, recent work [12] on nearest neighbour searches claims to accelerate PatchMatch by a factor of 10-20 in the $2 \mathrm{D}$ case, by using a kd-tree and dimensionality reduction. Extending these ideas to spatio-temporal patches presents new challenges, such as choosing a basis which is effective in terms of dimensionality reduction and computational cost.

\section{CONCLUSION}

We have shown that automatic video inpainting with global optimisation of a patch-based functional in reasonable execution times is possible, even for high resolution videos. By extending the PatchMatch algorithm to the case of spatio-temporal patches, we are able to provide a fast, useable video inpainting algorithm. Furthermore, we have proposed a fast, simple solution to the problem of oversmoothing of video inpainting results which is particularly problematic in the case of video textures. We have compared our results with those of [17] and [14] and found that our algorithm produces very similar results in an order of magnitude less time. This work should help to make experimentation with automatic, generic video inpainting easier and therefore make further progress in the domain possible.

\section{ACKNOWLEDGEMENTS}

The authors would like to express their thanks to Miguel Granados for his kind help and for answering their questions concerning his work.

\section{REFERENCES}

[1] M. Bertalmio, G. Sapiro, V. Caselles, and C. Ballester, "Image inpainting," SIGGRAPH, pp. 417-424, 2000.

[2] S. Masnou and J.-M. Morel, "Level lines based dissoclusion," ICIP, vol. 3, pp. 259-263, 1998.

[3] S. Masnou and J.-M. Morel, "Disocclusion: a variational 
approach using level lines," IEEE Trans. Image Processing, vol. 11 , no. 2, 2002.

[4] A. Criminisi, P. Perez, and K. Toyama, "Object removal by exemplar-based inpainting," CVPR, pp. 721-728, 2003.

[5] K. A. Patwardhan, G. Sapiro, and M. Bertalmio, "Video inpainting of occluding and occluded objects," ICIP, vol. 2, pp. 69-72, 2005.

[6] J. Jia, Y-W. Tai, T-P. Wu, and C-K. Tang, "Video repairing under variable illumination using cyclic motions," PAMI, vol. 28, no. 5, pp. 832-839, 2006.

[7] M. V. Venkatesh, S-C. S. Cheung, and J. Zhao, "Efficient object based video inpainting," ICIP, vol. 30, pp. 168-179, 2006.

[8] C-H. Ling, C-W. Lin, C-W. Su, Y-S. Chen, and H-Y. Liao, "Virtual contour guided video object inpainting using posture mapping and retrieval," IEEE Trans. Multimedia, vol. 13, no. 2, pp. 292-302, 2011.

[9] Y. Wexler, E. Shechtman, and M. Irani, "Space-time video completion," CVPR, vol. 1, pp. 120-127, 2004.

[10] Y. Pritch, E. Kav-Venaki, and S. Peleg, "Shift-map editing," ICCV, pp. 151-158, 2009.

[11] Y. Boykov, O. Veksler, and R. Zabih, "Fast approximate energy minimization via graph cuts," IEEE Trans. PAMI, vol. 23, no. 11, pp. 1222-1239, 1999.

[12] K. He and J. Sun, "Computing nearest-neighbor fields via propagation-assisted kd-trees," CVPR, pp. 111-118, 2012.

[13] Y. Liu and V. Caselles, "Exemplar-based image inpainting using multiscale graph cuts," IEEE Trans Image Process., vol. 22, pp. 1699-1711, 2013.

[14] M. Granados, J. Tompkin, K. Kim, O. Grau, J. Kautz, and C. Theobalt, "How not to be seen - object removal from videos of crowded scenes," Eurographics, vol. 31, pp. 219-228, 2012.

[15] G. Facciolo, R. Sadek, A. Bugeau, and V. Caselles, "Temporally consistent gradient domain video editing," EMMCVPR, vol. 6819, pp. 59-73, 2011.

[16] S. Darabi, E. Schechtman, and C. Barnes, "Image melding : Combining inconsistent images using patch-based synthesis," SIGGRAPH, vol. 31, 2012.

[17] Y. Wexler, E. Schechtman, and M. Irani, "Space-time completion of video," IEEE Trans. PAMI, vol. 29, no. 3, pp. 463-476, 2007.

[18] S. Arya and D. Mount, "Approximate nearest neighbor queries in fixed dimensions," SIAM, 1993.

[19] C. Barnes, E. Schechtman, A. Finkelstein, and D. B. Goldman, "Patchmatch: A randomized correspondence algorithm for structural image editing," SIGGRAPH, vol. 28, no. 3, 2009.

[20] "http://www.photoshopessentials.com/photo-editing/contentaware-fill-cs5/,"

[21] M. Granados, J. Tompkin, K. Kim, O. Grau, J. Katuz, and C. Theobalt, "How not to be seen - inpainting dynamic objects in crowded scenes," Tech. Rep., Max-Planck Institute für Informatik, February 2011. 